\title{
O Centro de Memória e Museu Histórico da Faculdade de Medicina de Ribeirão Preto: contribuições para a história e a educação médica
}

\section{The Center for Memory and Historical Museum at the Ribeirão Preto Medical School: contributions to history and medical education}

\author{
Roberta Vasconcelos \\ Leite ${ }^{i}$ \\ i Professora, Faculdade de Medicina/ \\ Universidade Federal dos Vales do \\ Jequitinhonha e Mucuri. \\ Diamantina - MG - Brasil \\ roberta.leite@ufvjm.edu.br \\ orcid.org/0000-0003-3110-0509
}

Recebido em 21 ago. 2019. Aprovado em 4 nov. 2019.
LEITE, Roberta Vasconcelos. O Centro de Memória e Museu Histórico da Faculdade de Medicina de Ribeirão Preto: contribuições para a história e a educação médica. História, Ciências, Saúde - Manguinhos, Rio de Janeiro, v.27, n.4, out.-dez. 2020, p.1355-1365.

\section{Resumo}

Problematizando o lugar da história e da memória na educação médica, apresentamos a trajetória de constituição e o acervo do Centro de Memória e Museu Histórico da Faculdade de Medicina de Ribeirão Preto. Reunindo objetos tridimensionais, fotografias, fitas cassete e coleções resultantes de doações, o centro pretende viabilizar pesquisas históricas e promover formação cultural dos estudantes, particularmente de medicina. A iniciativa afirma o potencial das humanidades para desenvolver espírito crítico e preserva rico acervo que testemunha criatividade dos pioneiros da pesquisa básica em medicina no Brasil. Concluímos que a experiência em curso na instituição carrega o potencial de contribuir para a história e a educação médica ao vincular-se à visão ampla de ciência e universidade.

Palavras-chave: educação médica; história; museus; acervos.

\section{Abstract}

This investigation of the role played by history and memory in medical education involves the establishment of the Center for Memory and Historical Museum at the Ribeirão Preto Medical School. By bringing together three-dimensional objects, photographs, cassette tapes, and collections of donated objects, the Center facilitates historical research and promotes the cultural formation of students, particularly in the area of medicine. This initiative affirms the function of the humanities in developing a critical spirit, and preserves a rich collection that bears witness to the creativity of Brazil's pioneers in medical research. We conclude that this institution has the potential to contribute to history and medical education through its connection to a broad vision of science and university.

Keywords: medical education; history; museums; collections.

http://dx.doi.org/10.1590/S0104-59702020000500017 
A educação médica no Brasil vivencia intensas transformações ao longo das últimas décadas. A partir do diagnóstico de que a formação dos médicos não atendia às necessidades da população e às demandas contemporâneas de saúde (Lampert et al., 2009), iniciou-se um processo de redimensionamento do modelo pedagógico dos cursos de graduação que culminou na publicação de novas Diretrizes Curriculares Nacionais (DCNs) em 2001 e 2014. Nesse contexto, reintroduzir a cultura humanística na formação tem se revelado estratégia que articula saberes e discursos, (re)criando formas de pensar e fazer medicina (Rios, 2010).

Diante desse cenário, perguntamos: seria possível inferir que a valorização da cultura por meio do cultivo da memória e da história pudesse favorecer a educação médica? Dada a atual projeção para o futuro e para a novidade, haveria lugar para a atenção aos percursos que levaram a profissão a ser como é hoje?

É certo que a disciplina história da medicina se firma há muito tempo como conteúdo obrigatório de todos os cursos e justifica sua pertinência para a compreensão da ciência e da profissão médicas ao oferecer perspectivas de sua evolução e tendências, alertando o estudante em relação à transitoriedade da verdade científica (Gusmão, 2004). Avançando nessa direção, Mota e Schraiber (2012) tratam de contribuições da história ao hodierno debate sobre a importância das humanidades no ensino médico, destacando como categorias de análise histórica mostram-se pertinentes para a construção do cuidado no encontro clínico, no qual os conhecimentos biomédicos se entrelaçam à história de vida do paciente e suas significações.

Em outra vertente, defensores da importância da memória e da história buscam garantir seu espaço no campo médico por meio da constituição de museus e centros de memória, bem como recorrendo à publicação de obras sobre a trajetória de instituições (Candeias, 1984; Teixeira, 2001). Analisando a evolução das escolas médicas ao longo dos séculos, é possível reconhecer que os museus, particularmente os de anatomia, já figuravam entre os serviços mais importantes oferecidos por essas instituições e eram cruciais para o ensino até o advento da medicina hospitalar e ambulatorial (Reinarz, 2005; Turk, 1994). Atualmente, não obstante o reconhecimento dos museus históricos como importante ferramenta pedagógica (Rodrigues, 2010), pode-se afirmar que os museus de medicina brasileiros não aproveitam seu potencial educativo porque funcionam com escassos recursos, dependem largamente do voluntariado de seus idealizadores e enfrentam enormes riscos de deterioração de seus acervos por falta de trabalho técnico ou condições adequadas de preservação (Serres, 2012).

Ainda assim - ou justamente em virtude desse contexto - são notórios os esforços dessas instituições em salvaguardar a memória, os registros e os equipamentos que contam a evolução da profissão no país. Em muitos museus de medicina, especialmente aqueles vinculados a instituições de ensino, busca-se hoje uma articulação entre conhecimento histórico e educação que se afaste dos modelos rígidos e lineares da "história oficial" (Mota, Marinho, 2007). Em lugar da mera exaltação institucional ou da identidade profissional, aposta-se em projetos voltados para a comunidade externa com vistas a difundir o conhecimento presente nos acervos e despertar vocação científica em crianças e adolescentes (Oliveira, Peret-Filho, 2017).

Alinhavando essas ponderações à discussão atual sobre a humanização na educação médica, retornam os questionamentos: numa profissão hoje tão voltada para o futuro 
com suas inovações e para o presente com seu apelo ao cuidado integral, há efetivamente tempo e espaço para o resgate histórico? Vale mesmo a pena insistir que o estudante de hoje, assoberbado com tantas exigências de conhecimento rigoroso e aplicação precisa de técnicas, olhe para o que já passou? Afinal, projetos voltados para o cultivo da memória e da história podem contribuir para uma educação médica crítica e humanizada ou se restringem a saudosismo dos antigos ou a incentivo ao conhecimento científico para adolescentes?

Tais questionamentos remetem ao incessante debate sobre a pertinência da história e da memória para os dias atuais. Debruçando-nos sobre o tema, realizamos pesquisa fenomenológica sobre a experiência de guardiões de memórias contemporâneos (Leite, 2015), a partir da qual nos vimos agora mobilizados a apresentar uma das iniciativas de preservação então estudadas, empreendida em um curso de medicina. Assim, no presente texto apresentamos a trajetória de constituição e o acervo do Centro de Memória e Museu Histórico $(\mathrm{CMMH})$ da Faculdade de Medicina de Ribeirão Preto da Universidade de São Paulo (USP), bem como apontamos desafios e contribuições que o cuidado com a memória pode oferecer à história da medicina no Brasil e à formação médica nos dias atuais.

\section{Caminhos e personagens da constituição do Centro de Memória e Museu Histórico}

Fundada em 1952, a Faculdade de Medicina de Ribeirão Preto (FMRP) integra a USP e foi pioneira no ensino médico no interior paulista. Sua constituição em meados do século XX é exemplo do processo de expansão universitária que almejava o desenvolvimento científico do país. A estreita relação com agências de fomento nacionais e internacionais, particularmente a Fundação Rockefeller, favoreceu a contratação de professores, a construção de laboratórios e o financiamento de pesquisadores e estudantes (Miranda et al., 2015).

É recorrente a menção à atuação de Zeferino Vaz, seu primeiro diretor, que se empenhou na garantia de instalações adequadas, formação do corpo docente com renomados ou promissores pesquisadores (em parte estrangeiros) e projeção nacional e internacional (Mauro, Nogueira, 2004). Já em sua primeira década de funcionamento, caracterizou-se por estruturas curricular e administrativa inovadoras, promovendo dedicação integral dos professores ao binômio ensino-pesquisa (Carlotti Junior, 11 out. 2014), bem como recebendo visitas de renomados cientistas da época, entre os quais ganhadores do prêmio Nobel: Alexander Fleming, Bernardo Houssay, Carl Schmitt, Corneille Heymans, Ragnar Granit e Severo Ochoa (Araújo, 2007). A tradição em pesquisa básica consolidou o renome internacional da instituição, em que se destacam trabalhos voltados para o diagnóstico e a profilaxia da doença de Chagas, capitaneados pelo professor José Lima Pedreira de Freitas, que fundou e organizou o Departamento de Higiene e Medicina Preventiva (Rocha, 2016); bem como a produção do professor Sergio Henrique Ferreira, que na década de 1960 descobriu a molécula BPF (fator de potenciação da bradicinina), possibilitando à indústria o desenvolvimento da classe de fármacos mais utilizados no mundo para o controle da pressão alta (Tuffani, 18 jul. 2016).

Outra figura crucial para a história da FMRP é Miguel Rolando Covian. Cientista argentino e discípulo de Houssay, transferiu-se para o Brasil a convite de Zeferino Vaz para estruturar o Departamento de Fisiologia. Considerado um dos mais importantes pesquisadores da neurofisiologia de sua época, foi responsável pela projeção internacional 
do departamento já em sua primeira década (Candido, Massimi, 2010). Seu interesse pela relação cérebro/mente por meio da relação entre fisiologia e comportamento foi decisivo para o desenvolvimento das neurociências no país (Miranda et al., 2015). Covian destacouse na história da FMRP também por sua personalidade, que aliava rigor científico à cultura humanística, encantando gerações de jovens que se formaram médicos e pesquisadores sob sua tutela (Massimi, Hoffmann, 2018). Para introduzir seu pensamento - essencial para compreensão da constituição do CMMH - vale a pena destacar trechos de um de seus escritos sobre a função da universidade:

É função humanística da Universidade proporcionar a cada geração a possibilidade de conhecer, refletir e avaliar a herança cultural que recebeu. Que o estudante possa mergulhar nas riquezas da cultura a fim de que se desperte nele o desejo de viver a aventura de uma vida orientada pela Verdade, a Bondade e a Beleza, que constituem nossa herança humana. ... Cumpre-lhe formar o homem total e não irrisórias caricaturas, parcelas de um quebra-cabeça, no fundo parcelas humanas. Não se podem e não se devem modelar mentes e espíritos de acordo com um plano determinado, que pode ser bom para certos interesses, mas não para a formação humanística, cuja tarefa central é entender o homem e dar-lhe possibilidades para desenvolver aquilo que faz digna a vida (Covian, 2007, p.67-69).

A envergadura da produção científica e contribuição ao ensino médico da FMRP mobilizou a publicação de diversas obras (Araújo, 2007; Mauro, Nogueira, 2004; Rodrigues et al., 2018), bem como a preocupação em reunir e salvaguardar seu patrimônio documental. Nesse sentido, já em 1985 o então acadêmico Osvaldo Cardoso Santana Filho sugere a instalação do Acervo Histórico da FMRP, e, no mesmo ano, a Congregação constitui uma comissão de criação do órgão presidida pelo professor Ruy Escorel Ferreira Santos. Equipamentos e documentos começam a ser reunidos, mas a iniciativa perde força e é retomada somente na década seguinte (Museu Histórico..., s.d.).

No início da década de 1990, Anette Hoffmann, professora do Departamento de Fisiologia, passa a coordenar as atividades da Assessoria Cultural da FMRP - futuramente instituída como Comissão de Cultura e Extensão, também presidida por ela -, entre outras razões, por sua atuação na organização de exposições de arte no saguão do prédio central, as quais tiveram início ainda na gestão do professor Dalmo Amorim como diretor. Logo ao iniciar os trabalhos, foi alertada pela funcionária Maria Alice Coelho que em uma das salas da área administrativa havia documentos reunidos em 1985 por alguns professores, entre eles Covian e Ruy Ferreira Santos: desenhos que foram feitos para desenvolver o brasão da faculdade; recortes de jornais da época em que a faculdade foi criada; muitas fotografias dos primeiros anos de funcionamento. Assim começava a ser retomado o material daqueles que haviam se disposto a reunir a história da FMRP anos antes. Em depoimento presente em Leite (2015, p.102), Anette afirma: "a partir do momento em que soube desse material pensei: como presidente da Comissão, não posso deixar isso passar sem tomar a iniciativa de tentar concretizar a ideia desses professores".

Ao referido material já reunido pelos professores, somava-se outro rico acervo que estava aos cuidados dela, ainda sem destinação adequada. Trata-se de correspondências, documentos, fotografias, livros e vinis do professor Covian, pois, após seu falecimento 
em 1992, a FMRP recebeu como doação tudo o que estava em seu gabinete e também a biblioteca que ficava em sua casa. Em virtude dos muitos anos de convivência e trabalho em conjunto, Anette foi encarregada de selecionar e dar destinação ao material. Logo percebeu que ele havia guardado de forma bem ordenada as correspondências, principalmente as cartas trocadas com Houssay, que foi o seu mestre. Além das recebidas, havia cópias carbono das que foram enviadas. Essa correspondência denota que, nos árduos primeiros anos de permanência em Ribeirão Preto, a figura de Houssay foi de extrema importância para Covian, tanto por mantê-lo ligado às origens quanto por prover força para dar continuidade ao trabalho na FMRP (Leite, 2015).

Quanto à biblioteca e aos vinis de Covian, também doados ao departamento, a biblioteca central da FMRP não aceitou receber o material por seu caráter eclético, com poucos livros "científicos" e muitos de teologia, filosofia e arte. Esse material ficou por um tempo "esquecido" em um corredor, tendo sido alvo de furtos e deterioração até que pudesse ser reunido ao acervo do nascente Centro de Memória e Museu Histórico, integrando a coleção "Miguel Rolando Covian" (Leite, 2015).

Com o desenvolvimento do projeto do CMMH, foi possível também salvar do descarte equipamentos dos primeiros anos da FMRP, construídos na Oficina Mecânica de Precisão do campus por técnicos e professores como Ricardo Marseillan e José Venâncio Pereira Leite. Professores que, com engenhosidade e peças simples, dedicavam-se a idealizar equipamentos específicos para experimentos ali desenvolvidos nas primeiras décadas da faculdade, por exemplo, estimuladores elétricos, amplificadores para registros de eventos como potenciais cerebrais e medidores de fluxo de sangue.

Em 1997, documentos e equipamentos foram reunidos no primeiro espaço cedido pela faculdade: ${ }^{1}$ a antiga sede do Centro Acadêmico, situada onde outrora os pesquisadores de parasitologia acomodavam as galinhas contaminadas com doença de Chagas. Inicia-se então um longo processo de diligências com vistas a garantir espaço e instalações adequados aos objetivos do museu que, após esse primeiro local, ocupou uma das antigas residências de professores no campus até finalmente - por ação do então diretor da FMRP, professor Marcos Felipe Sousa Sá - conquistar seu lugar no Espaço de Cultura e Extensão Universitária (Eceu), na avenida Nove de Julho, região central de Ribeirão Preto (Museu Histórico..., s.d.). Investigações realizadas no acervo do CMMH já resultaram em trabalhos de conclusão de curso, dissertações de mestrado, pesquisa de pós-doutorado (Bueno, Massimi, 2007; Candido, Massimi, 2010; Damasceno, 2013; Miranda et al., 2015; Pizella, Massimi, 2007).

Ao longo dos anos, Anette Hoffmann permanece à frente da constituição e manutenção do CMMH (Massimi, 2018). Cuidando ativamente da conservação do legado dos fundadores da FMRP, angariou fundos para a compra de equipamentos adequados à conservação de parte do acervo e para a capacitação da equipe e publicou diversos trabalhos sobre a experiência universitária de seu mestre Covian (Hoffmann, 2005, 2007; Miranda et al., 2015). Em parceria com a professora e historiadora Marina Massimi, ${ }^{2}$ tem se dedicado a promover encontros entre diferentes áreas do saber por meio de cursos, exposições e publicações (Hoffmann, Bueno, Massimi, 2001; Hoffmann, Massimi, 2007, 2008, 2011).

Como exemplo, citamos os projetos "Reflexões em torno da dor", "Ciência: da maravilha à descoberta" e, em especial, "Polifonias do coração". Neste último, o coração humano foi 
contemplado a partir da perspectiva de médicos, historiadores, filósofos, músicos, poetas, teólogos. Formalizado como curso de extensão, disciplina eletiva em dois programas de pós-graduação da USP e exposição temporária no CMMH em 2011, foi também publicado como livro (Hoffmann, Oliveira, Massimi, 2014). Destaca-se ainda a articulação com o Museu de Arte de Ribeirão Preto, por meio do Projeto Medicina e Arte, com exposições organizadas no CMMH que atraem público diversificado (Jesus, 25 out. 2016).

Hoje, como professora colaboradora sênior, Anette Hoffmann tem assento no Conselho Deliberativo do CMMH e integra o grupo de pesquisa "Tempo, memória e pertencimento", do Instituto de Estudos Avançados da USP, coordenado por Marina Massimi. Recentemente, o grupo organizou o evento "Miguel Rolando Covian: uma concepção de universidade para os tempos atuais", em que diversos ex-alunos, ${ }^{3}$ atualmente professores universitários, narraram a influência de Covian em suas trajetórias acadêmicas (Massimi, Hoffmann, 2018).

Para organização do acervo, monitoria a visitas programadas e a oficinas periodicamente oferecidas ao público, e divulgação em sites e redes sociais, ${ }^{4}$ o CMMH já contou com um funcionário exclusivo, além dos estagiários e bolsistas mantidos pela Pró-Reitoria de Cultura e Extensão Universitária da USP (Sobre o museu, 2011). Em 2019, como mais um exemplo das contenções de gastos que afetam todas as universidades públicas brasileiras, o único funcionário encontra-se deslocado para outro setor e os cortes de verbas ameaçam a adequada manutenção do acervo.

\section{O acervo do Centro de Memória e Museu Histórico da Faculdade de Medicina de Ribeirão Preto}

Ainda que não esteja devidamente catalogado, em virtude da carência de profissionais qualificados, o inventário a seguir demonstra como o acervo do $\mathrm{CMMH}$ conta com números expressivos:

- 242 objetos tridimensionais, como instrumentos e equipamentos de uso em pesquisa, ensino e atendimento clínico/hospitalar, utilizados na FMRP entre as décadas de 1950 e 1970. Desses, 210 foram devidamente identificados e catalogados, sendo que oitenta foram desenvolvidos na Oficina Mecânica de Precisão da FMRP e constituem réplicas de objetos importados ou soluções autóctones para problemas apresentados por docentes.

- 1.581 cópias de fotografias e 390 mil negativos fotográficos (em base plástica e em chapas de vidro). As cópias fotográficas foram digitalizadas e organizadas em pastas separadas por assuntos como: eventos, laboratórios, assistência hospitalar, visitantes (incluindo as de membros da Fundação Rockefeller). Os negativos pertenceram ao Serviço de Documentação Científica da FMRP e se referem a solicitações de docentes interessados em documentar casos clínicos e cirúrgicos ou trabalhos experimentais.

- 146 fitas cassete, com gravações de depoimentos de professores, funcionários e alunos que vivenciaram os anos iniciais da FMRP, realizadas pelos historiadores José Eduardo Marques Mauro e Arlinda Rocha Nogueira entre 1990 e 1993. Alguns desses depoimentos foram transcritos e todos foram transpostos para pen drive. 
- 3 coleções resultantes de doações

- Coleção Miguel Rolando Covian, doada pelo Departamento de Fisiologia da FMRP. Formada por: 1.500 livros de assuntos diversos como filosofia, teologia, ciências e literatura, mostrando a vasta formação cultural do professor; 200 discos de vinil, sobretudo de música erudita; artigos científicos e capítulos de livros publicados em revistas e livros nacionais e estrangeiros; artigos sobre assuntos culturais (por exemplo, concepção de universidade, importância da formação cultural do estudante, relação entre ciência e religião); fotografias e diapositivos; 1.546 correspondências, com destaque para as cartas trocadas entre Covian e Houssay (217 recebidas de seu mestre e 194 cópias daquelas que lhe enviou).

- Coleção Pedreira de Freitas, doada pela família do professor José Lima Pedreira de Freitas. Formada por aproximadamente 4.850 separatas de autoria de diversos pesquisadores, que trabalhavam na mesma linha de pesquisa ou em linhas próximas; 5 instrumentos e equipamentos usados em pesquisa e campanhas de erradicação da doença de Chagas; 1 memorial e tese de cátedra.

- Coleção Fritz Koeberle, doada pela família desse professor austríaco que, a convite de Zeferino Vaz, mudou-se para Ribeirão Preto para organizar o Departamento de Patologia. Destacou-se por suas pesquisas em doença de Chagas. A coleção conta com 148 livros, a maioria em idioma alemão; separatas e capítulos de livros de sua autoria, publicados tanto no Brasil quanto no exterior; 148 negativos fotográficos; 1.469 diapositivos com imagens científicas, provavelmente usados em aulas ou conferências.

Com esse breve inventário é possível vislumbrar o potencial do material existente para estudos históricos sobre: a interiorização do ensino superior público no estado de São Paulo; o paradigma de escola médica voltada para a pesquisa básica que a FMRP inaugurou no país; a atuação da Fundação Rockefeller na faculdade e seu papel na criação desse novo paradigma.

Além disso, a coleção de objetos tridimensionais - particularmente os de fabricação local - é representativa de um período que precedeu a introdução da tecnologia digital na área médica. Quanto às coleções, duas delas podem subsidiar pesquisas sobre o estudo da doença de Chagas no Brasil e o protagonismo da FMRP nesse campo.

Já a coleção Covian, além de ter fomentado a realização das pesquisas já mencionadas - com foco em relacionamentos, trajetória e pensamentos do cientista (Bueno, Massimi, 2007; Candido, Massimi, 2010; Damasceno, 2013; Miranda et al., 2015; Pizella, Massimi, 2007) - tem potencial para investigações sobre a ligação da faculdade à escola de fisiologia argentina liderada por Houssay e a formação de uma rede de colaborações internacionais num momento em que a distância dos centros culturais poderia ser prejudicial à jovem faculdade.

\section{Discussão: o potencial da memória e da história para a educação médica}

Após percorrer a trajetória de constituição e conhecer o acervo do CMMH da FMRP, é fácil perceber que o centro de memória e os projetos a ele associados fazem eco ao movimento de valorização da cultura humanística na formação em medicina (Gallian, 
2000; Guardiola, Baños, 2017; Rios, 2010). Frente ao excesso de tecnicismo e desumanização das condutas profissionais, tem-se um espaço que documenta outras formas possíveis de fazer ciência e educar médicos. Escolhendo sobretudo temas que são necessariamente interdisciplinares - como a vivência da dor ou a compreensão sobre os significados do coração para a humanidade -, os projetos desenvolvidos no CMMH denotam um caminho particularmente fecundo para alicerçar a constatação de que a medicina não pode sobreviver enclausurada em si mesma.

Projetos como esses seriam capazes de reverter a pouca valorização e o baixo aproveitamento do potencial pedagógico dos museus e centros de memória da medicina, denunciados por Serres (2012)? Pesquisas prévias (Leite, 2015; Leite, Mahfoud, 2018a, 2018b) nos indicam que o potencial dos museus e espaços dedicados à preservação da memória não está apenas em seus acervos ou nos projetos empreendidos, mas no tipo de experiência que motivou sua constituição. Considerando a trajetória do $\mathrm{CMMH}$, reconhecemos uma visão ampla de ciência e universidade como grande força motriz dos esforços de criação e manutenção da instituição. Entendemos ser essa visão o que a torna potente testemunha de um caminho fecundo - que atualmente parece a muitos impossível - no qual exigências de rigor científico da pesquisa básica aliam-se à criatividade, à liberdade intelectual e ao desejo de formar médicos que saibam reconhecer o valor da cultura e sejam capazes de "desenvolver aquilo que faz digna a vida" (Covian, 2007, p.69).

Como os demais museus universitários - e museus em geral -, o CMMH enfrenta o desafio de superar enfoques bairristas e saudosistas na apresentação dos acervos de modo a despertar fascínio e instigar acadêmicos a se interessar pelas heranças que gerações anteriores lhes legaram e delas cuidar. Nessa difícil equação, faz-se necessário equilibrar as motivações pessoais de quem se dedica a preservar a memória, as necessidades técnicas de preservação do material histórico e a importância de sistematização dos registros para que possam subsidiar investigações. Para tanto, o CMMH tem buscado unir a vitalidade pessoal de seus mantenedores e o interesse pela pesquisa à solidez institucional. Tarefa árdua num contexto como o atual, em que cortes de verbas se somam ao menosprezo à história e à produção científica universitária.

A despeito dos ventos desfavoráveis, o CMMH da FMRP segue de portas abertas oferecendo aos interessados o acesso a um rico acervo com grande potencial para realização de pesquisas que contribuam para o avanço do conhecimento da história da medicina e da ciência brasileiras. Ainda há um longo percurso a trilhar em termos de mobilização para que graduandos e pós-graduandos da faculdade se dediquem a pesquisas em seu âmbito. Os obstáculos que enfrentam, porém, parecem não destituir o valor e a força da iniciativa, que se assenta na potente articulação entre o cuidado vitalizado e o rigor científico e histórico.

Num momento em que a preocupação histórica parece tão distante do exigente cotidiano dos acadêmicos, a trajetória de constituição e o acervo do CMMH testemunham ser imprescindível uma concepção ampla de formação e universidade para justificar - na educação médica e universitária em geral - a dedicação à cultura, à interdisciplinaridade, ao passado. 


\section{NOTAS}

${ }^{1}$ A cessão da área física foi aprovada em 13 de dezembro de 1996 pelo Conselho Técnico-Administrativo da FMRP.

${ }^{2}$ Referência nacional e internacional no campo da história da psicologia, Marina Massimi atuou, tal como Anette, na Faculdade de Filosofia, Ciências e Letras, no campus Ribeirão Preto da USP. Professora titular aposentada, tornou-se colaboradora sênior e hoje lidera grupo de pesquisa "Tempo, memória e pertencimento" no Instituto de Estudos Avançados da USP.

${ }^{3}$ Além da própria Anette, contribuíram com relatos os professores Paulo Saldiva (diretor do Instituto de Estudos Avançados da USP); Marta Edna Holanda Diogenes Yazlle e José Antunes Rodrigues (FMRP/USP) e Luis Menna Barreto (Instituto de Ciências Biomédicas e Escola de Artes, Ciências e Humanidades/USP). No depoimento deste último destaca-se a narrativa sobre como, mesmo após sua prisão política pelo envolvimento no movimento estudantil nos anos da ditatura, foi acolhido por Covian para realização de suas pesquisas sobre neurociências na pós-graduação da FMRP.

${ }^{4}$ Cf. o perfil no facebook <https://pt-br.facebook.com/museufmrpusprp/>, o blog <http://museufmrpusp. blogspot.com/> e a página sobre o museu no site institucional da FMRP <https://www.fmrp.usp.br/ institucional/marcos-historicos/museu-historico $>$.

\section{REFERÊNCIAS}

ARAÚJO, Marcelo José.

A Faculdade de Medicina de Ribeirão Preto, USP (1948-1975). Dissertação (Mestrado em Educação) - Universidade Federal de São Carlos, São Carlos. 2007.

BUENO, Patrícia Moura de Souza; MASSIMI, Marina.

A experiência universitária na correspondência epistolar entre Miguel Rolando Covian e Bernardo Houssay. In: Hoffmann, Anette; Massimi, Marina (Org). A universidade pensada e vivida por Miguel Rolando Covian. Ribeirão Preto: Funpec. p.28-44. 2007.

CANDEIAS, Nelly Martins Ferreira.

Memória histórica da Faculdade de Saúde Pública da Universidade de São Paulo: 1918-1945. Revista de Saúde Pública, v.18, n.esp., p.2-60. 1984.

CANDIDO, Marcos; MASSIMI, Marina.

Perspectivas para uma formação humanística do estudante universitário no pensamento de Miguel Rolando Covian. Memorandum, v.19, p.210-224. 2010.

CARLOTTI JR., Carlos Gilberto.

Medicina de Ribeirão Preto: 62 anos de reconhecimento no país e no exterior. O Estado de São Paulo, 11 out. 2014. Disponível em: <https:// sao-paulo.estadao.com.br/noticias/geral,medicinade-ribeirao-preto-62-anos-de-reconhecimento-nopais-e-no-exterior-imp-,1575259>. Acesso em: 8 set. 2018. 11 out. 2014

COVIAN, Miguel Rolando.

Cultura humanística do estudante universitário. In: Hoffmann, Anette; Massimi, Marina (Org.). A universidade pensada e vivida por Miguel Rolando Covian. Ribeirão Preto: Funpec. p.64-73. 2007.
DAMASCENO, Eneida Nogueira.

Criação de arquivo epistolar do neurofisiologista Miguel Rolando Covian: um registro históricocontextual. Dissertação (Mestrado em Psicologia) - Universidade de São Paulo, Ribeirão Preto. 2013

GALLIAN, Dante Marcello Claramonte. A (re)humanização da medicina. Psiquiatria na Prática Médica, v.33, n.2, p.1-5. 2000.

GUARDIOLA, Elena; BAÑOS, Josep. El papel de las humanidades médicas en la educación de los profesionales de la salud del siglo XXI. Revista de Medicina y Cine, v.13, n.4, p.155-157. 2017.

GUSMÃO, Sebastião.

História da medicina: evolução e importância. Jornal Brasileiro de Neurocirurgia, v.15, n.1, p.5-10. 2004.

HOFFMANN, Anette.

A personalidade científica e humana de Miguel Rolando Covian (1913-1992). In: Hoffmann, Anette; Massimi, Marina (Org). A universidade pensada e vivida por Miguel Rolando Covian. Ribeirão Preto: Funpec. p.1-7. 2007.

HOFFMANN, Anette.

Resgate da memória de uma experiência universitária: a história de Miguel Rolando Covian. Congresso Paulista de História da Medicina, I, Ribeirão Preto. In: Anais... Ribeirão Preto: FMRP. 2005.

HOFFMANN, Anette; BUENO, José Lino de Oliveira; MASSIMI, Marina (Org.).

Percorrer distâncias: um desafio para a razão humana. São Paulo: Companhia Ilimitada. 2001. 
HOFFMANN, Anette; MASSIMI, Marina (Org.). Ciência: da maravilha à descoberta. Ribeirão Preto: Funpec. 2011.

HOFFMANN, Anette; MASSIMI, Marina (Org.). Reflexões em torno da dor. Ribeirão Preto: São Francisco Grupo Gráfico. 2008.

HOFFMANN, Anette; MASSIMI, Marina (Org.). A universidade pensada e vivida por Miguel Rolando Covian. Ribeirão Preto: Funpec. 2007.

HOFFMANN, Anette; OLIVEIRA, Leda Menescal de; MASSIMI, Marina (Org.).

Polifonias do coração. Ribeirão Preto: Funpec. 2014.

JESUS, André Luís de.

Reportagem sobre o Museu Histórico da Faculdade de Medicina de Ribeirão Preto. TV Câmara Ribeirão Preto. 25 out. 2016.

LAMPERT, Jadete Barbosa et al. Projeto de avaliação de tendências de mudanças no curso de graduação nas escolas médicas brasileiras. Revista Brasileira de Educação Médica, v.33, supl.1, p.5-18. 2009.

LEITE, Roberta Vasconcelos.

Experiência ontológica e tradição na experiência de guardiões de memórias contemporâneos. Tese (Doutorado em Psicologia) - Universidade Federal de Minas Gerais, Belo Horizonte. 2015.

LEITE, Roberta Vasconcelos; MAHFOUD, Miguel. $\mathrm{Da}$ arte invisível ao enraizamento: uma experiência de educação não formal no Subúrbio Ferroviário de Salvador. Revista Brasileira de Educação, v.23, p.e230087. 2018a.

LEITE, Roberta Vasconcelos; MAHFOUD, Miguel. Elaborando tradições na contemporaneidade: Ausier e a preservação do chorinho. Revista da Abordagem Gestáltica, v.24, n.2, p.145-156. 2018b.

MASSIMI, Marina.

Abertura. In: Miguel Covian: uma concepção de universidade para os tempos atuais. São Paulo: Instituto de Estudos Avançados da USP. Disponível em: <https://www.youtube.com/ watch? $\mathrm{v}=$ KoNLzloj6V4 $>$. Acesso em: $12 \mathrm{mar}$. 2019. 2018.

MASSIMI, Marina; HOFFMANN, Anette (Org.). Miguel Rolando Covian: uma concepção de universidade para os tempos atuais. São Paulo: Instituto de Estudos Avançados da USP. Disponível em: <http://www.iea.usp.br/eventos/ miguel-covian>. Acesso em: 12 mar. 2019. 2018.

MAURO, José Eduardo Marques; NOGUEIRA, Arlinda Rocha (Org.).

Faculdade de Medicina de Ribeirão Preto, USP: primeiros tempos, através dos documentos e pela voz de seus construtores. Ribeirão Preto: Funpec. 2004.
MIRANDA, Rodrigo et al.

Introducing Miguel Rolando Covian: humanism and scientific training in Brazil. Revista Peruana de Historia de la Psicologia, v.1, p.29-49. 2015.

MOTA, André; MARINHO, Maria Gabriela Silva Martins da Cunha.

Concepções de História e trajetórias

institucionais. Museu Histórico da Faculdade de Medicina da Universidade de São Paulo: análise e crítica de uma experiência (1977-2008).

Cadernos de História da Ciência, v.3, n.2, p.123143. 2007.

MOTA, André; SCHRAIBER, Lilia Blima.

Ciências humanas e medicina: as contribuições da história para a formação e a prática do médico. Revista de Medicina (São Paulo), v.91, n.3, p.189-193. 2012.

MUSEU HISTÓRICO...

Museu Histórico - Faculdade de Medicina de Ribeirão Preto. Disponível em: <http://www. fmrp.usp.br/institucional/marcos-historicos/ museu-historico >. Acesso em: 12 mar. 2019. s.d.

OLIVEIRA, Carla Cristina Vieira de; PERETFILHO, Luciano Amedée.

Organização e automação de acervo do Centro de Memória da Faculdade de Medicina. RACIn, v.12, n.2, p.136-146. 2017.

PIZELLA, Denise Gallo; MASSIMI, Marina. Universidade: essência, impasses e caminhos nos escritos de Miguel Rolando Covian. In: Hoffmann, Anette; Massimi, Marina (Org.). Universidade pensada e vivida por Miguel Rolando Covian. Ribeirão Preto: Funpec. p.8-27. 2007.

REINARZ, Jonathan.

The age of museum medicine: the rise and fall of the medical museum at Birmingham's School of Medicine. Social History of Medicine, v.18, n.3, p.419-437. 2005.

RIOS, Izabel Cristina.

Humanidades e medicina: razão e sensibilidade na formação médica. Ciencias e Saúde Coletiva, v.15, supl., p.1725-1732. 2010.

ROCHA, Juan Stuardo Yazlle.

José Lima Pedreira de Freitas e o redimensionamento e controle da doença de Chagas. Ciência e Saúde Coletiva, v.21, n.8, p.2631-2639. 2016.

RODRIGUES, Ana Ramos.

O museu histórico como agente de ação educativa. Revista Brasileira de História e Ciências Sociais, v.2, n.4, p.215-222. 2010.

RODRIGUES, Maria de Lourdes Veronese et al. (Org.).

Faculdade de Medicina de Ribeirão Preto, USP: primeiras décadas. Ribeirão Preto: Funpec. 2018. 
SERRES, Juliane Conceição Primon.

As redes de museus: preservação e difusão do patrimônio cultural da medicina no Brasil. Museologia e Patrimônio, v.5, n.1, p.145-155. 2012.

SOBRE O MUSEU.

Sobre o Museu. Disponível em: <http:// museufmrpusp.blogspot.com/p/o-museu.html $>$. Acesso em: 8 set. 2018. 2011.

TEIXEIRA, Rodolfo.

Memória histórica da Faculdade de Medicina do

Terreiro de Jesus: 1943-1995. Salvador: Edufba. 2001.
TUFFANI, Maurício.

Sérgio Henrique Ferreira (1934-2016): premiado cientista descobridor de remédios. Folha de S.Paulo, p.1-4. 18 jul. 2016.

TURK, John L.

The medical museum and its relevance to modern medicine. Journal of the Royal Society of Medicine, v.87, p.40-42. 1994. 